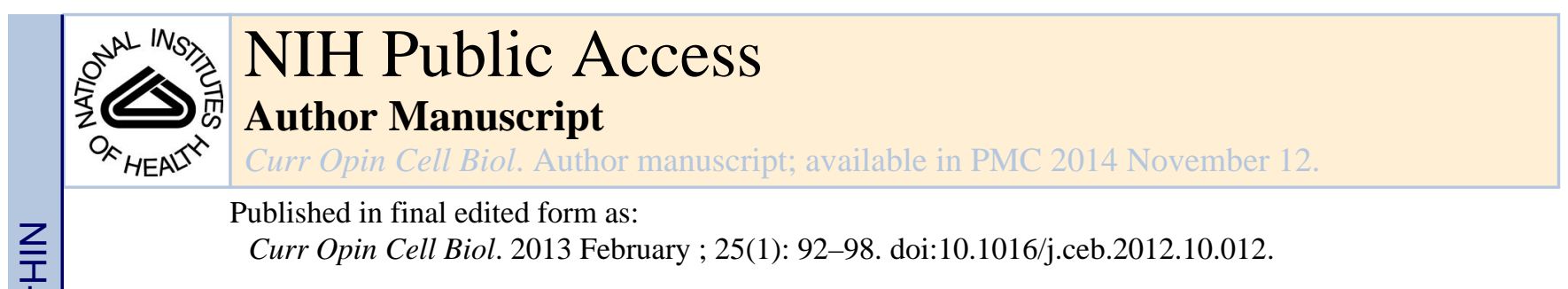

\title{
Membrane Dynamics during Cytokinesis
}

\author{
John A. Schiel and Rytis Prekeris ${ }^{1}$ \\ Department of Cell and Developmental Biology, School of Medicine, Anschutz Medical Campus, \\ University of Colorado Denver, Aurora, CO, 80045, USA
}

\begin{abstract}
Endocytic membrane transport has recently emerged as a key process required for the successful completion of cytokinesis. Specific endocytic membranes act in concert with the cytoskeleton and ESCRT proteins to regulate the various stages of cytokinesis. In this review, we focus on the different endocytic Arf and Rab GTPases and their interaction proteins that regulate organelle transport to the intracellular bridge during cytokinesis. The identity and function of these endocytic organelles during the late stages of cell division will also be discussed.
\end{abstract}

\section{Introduction}

The end of the cell cycle culminates in a very complex and highly orchestrated physical separation of daughter cells, known as cytokinesis. It was initially thought that plant and animal cells divide by completely different means. Plant cells divide by building a new cell wall via delivering membranes to a midzone-localized organelle, known as the phragmoblast. In contrast, animal cells were thought to divide solely by using their actomyosin contractile ring, without the need of new membrane delivery. Recent studies clearly demonstrate that membrane transport and subsequent fusion is an important step during cytokinesis in animal cells. Many studies from multiple organisms have shown that membranes originating from the Golgi apparatus and endosomes are trafficked to the intracellular bridge (ICB) of dividing cells and are required for both early and late steps of cytokinesis. The events comprising early cytokinesis have been the subject of many reviews [1-7] and will not be covered at length here. We will focus on membrane transport and its functional significance in regulating late cytokinesis.

\section{Regulation of membrane transport to the intracellular bridge}

While membrane transport has been shown to be important in regulating the early phases of cytokinesis, the identity and spatiotemporal properties of identified organelles during late cell division remain unclear [8-11]. Membrane trafficking during cytokinesis was initially investigated in C. elegans and Drosophila embryos by treatment with brefeldin A (BFA) to

(C) 2012 Elsevier Ltd. All rights reserved.

${ }^{1}$ Corresponding author (Rytis.Prekeris@ucdenver.edu).

Publisher's Disclaimer: This is a PDF file of an unedited manuscript that has been accepted for publication. As a service to our customers we are providing this early version of the manuscript. The manuscript will undergo copyediting, typesetting, and review of the resulting proof before it is published in its final citable form. Please note that during the production process errors may be discovered which could affect the content, and all legal disclaimers that apply to the journal pertain. 
inhibit anterograde transport from the endoplasmic reticulum (ER) to the Golgi apparatus, and from the Golgi apparatus to the plasma membrane (PM) (we will refer to this pathway as the secretory pathway) [12]. It was determined that this secretory pathway is important for cytokinesis, as BFA treatment caused regression of the ICB in C. elegans [13] and Drosophila embryos [14]. Additionally, Gromley et al. [8] and Goss and Toomre [15] both used fluorescently-tagged secretory markers, luminal-GFP and VSVG-YFP respectively, to further establish that secretory vesicles are transported to the ICB during cytokinesis where they fuse with the PM. However, in some experimental models (e.g. the sea urchin embryo), inhibition of the secretory pathway via BFA had no effect on cytokinesis [16]. Furthermore, it was shown that while secretory vesicles are present at the ICB during early telophase, they are largely absent during the abscission step of cytokinesis [11,17]. These results suggest that the secretory pathway may not be the key contributor of membrane during late cytokinesis, and that there must be additional trafficking pathways that are important during cell division.

The importance of recycling endosomes during cytokinesis was originally suggested from work studying the cellularization of fly embryos $[18,19]$. Subsequently, it was shown that recycling endosomes are important for cytokinesis in mammalian cells [20] and that these endosomes contain Rab11, a small monomeric GTPase required for late cytokinesis [18-20]. In a series of studies, it was discovered that Rab11-binding proteins FIP3 and FIP4 (Rab11 Family of Interacting Proteins 3 and 4) localize to the ICB and simultaneously interact with Rab11 as well as another small GTPase, Arf6 [20-22] (Table 1). Spatiotemporal analysis of FIP3-containing endosomes during cytokinesis demonstrated that FIP3-endosomes are a specific subpopulation of recycling endosomes, are delivered to the ICB during late telophase, and are required for generating a secondary ingression of the ICB to allow for the successful completion of abscission $[11,20,23,24]$. Finally, it was identified that the FIP3endosome cargo proteins, SCAMP2/3 and p50RhoGAP, have roles in depolymerizing cortical F-actin in the ICB prior to generation of the secondary ingression [23]. p50RhoGAP presumably achieves decrease in F-actin by inactivating Rho/Rac small GTPases, while the mechanism for SCAMP2/3 remains unclear [23].

Echard and colleagues [9] performed siRNA analysis of 29 different Rab GTPases and found that, in addition to Rab11, Rab35 is also required for cytokinesis (Table 1). Rab35 endosomes were shown to be important to maintain the stability of the ICB during cytokinesis and abscission [9] and to influence the localizations of two components known to be required for cytokinesis, phosphatidylinositol-4-5-biphosphate $\left(\operatorname{PtdIns}(4,5) \mathrm{P}_{2}\right)$ and SEPT2 [9]. Very recently, a PtdIns(4,5) $\mathrm{P}_{2}$ 5-phosphatase, oculocerebrorenal syndrome of Lowe (OCRL), was identified as a Rab35-binding protein that controls the hydrolysis of PtdIns $(4,5) \mathrm{P}_{2}$, which provides localized remodeling of the cortical actin network within the ICB [25].

While Rab11 and Rab35 are now well-established regulators that play a key role in cytokinesis, other Rab GTPases such as Rab4, Rab8, Rab14, and Rab21 are also present within either the midzone or the ICB (Table 1) [10,26-28]. Interestingly, none of these GTPases seem to be absolutely required for the completion of cytokinesis, suggesting that they may only play a modulatory role during cell division. Briefly, Rab4, a marker for early 
endosomes, was found within the central spindle where it co-localizes with Arf6, although the knock-down of Rab4 did not result in a cytokinesis defect [26]. Similarly, Rab8 was shown to localize to the ICB during early cytokinesis [10]. Recently, Dr. Reiner and colleagues [29] demonstrated that Rab8 interacts with cytoplasmic dynein and doublecortin domain-containing protein 5 (DCDC5). DCDC5 is a microtubule-associated protein that, when knocked-down, impairs cytokinesis and Rab8-vesicle positioning in the ICB [29]. However, the depletion of Rab8 does not lead to cytokinesis defects [9] and Rab8containing organelles are not present within the ICB during abscission [23,30]. Just like Rab4 and Rab8, Rab14 also localizes to endosomes during interphase and is present at the ICB during cytokinesis [27], although the requirement of Rab14 for cell division remains to be demonstrated. Rab21 was recently shown to be involved in the targeted trafficking of integrins into the ICB and that this activity is required for proper cytokinesis, at least in some adherent cell lines [28].

\section{The roles of endosomal membrane transport during cytokinesis}

Several studies in a variety of organisms have demonstrated that multiple Rab GTPases define specific subpopulations of endocytic organelles that are differentially transported to the ICB and presumably play distinct roles during cell division. To date, there has not been a comprehensive localization analysis to determine the spatio-temporal dynamics of various endocytic Rabs. As evidenced by recent data (Table 1), specialized populations of organelles are transported to the ICB via different pathways (Figure 1). However, we do not know if and when these endosomes are present at the ICB and the roles that these endosomes play during cytokinesis are only beginning to emerge. The rest of this mini-review will focus on describing the most recent findings about the functions of these different endosomes during cytokinesis.

\section{Membrane fusion is required for cytokinesis}

At the beginning of mitosis, during prophase and metaphase, cells round up resulting in the dramatic reduction of PM surface area [31]. This reduction is caused, at least in HeLa cells, by a decrease in recycling endosome fusion with the PM, without any change in the rate of endocytosis [31]. At the completion of anaphase, recycling endosome transport and fusion with the PM resumes, leading to the rapid recovery of daughter cell PM surface area. This regulated change in recycling endosome fusion with the plasma membrane appears to be required for mitosis, since its inhibition leads to cytokinesis defects [31,32]. Additional work has established a similar requirement for endosome fusion with the PM during cytokinesis in multiple other cell lines and organisms [16,33-35]. While it remains incompletely understood why cells need endosomal transport and fusion during cytokinesis, two possible explanations have been put forward regarding the roles of endosomes during division. First, it was suggested that endosomes may provide new membrane and/or specific lipids to the forming ICB. Second, endosomes may allow fast and localized delivery of regulatory proteins leading to the reorganization of the cytoskeleton and PM that is required for abscission to take place. In this mini-review we will not discuss the roles of specific lipids during cytokinesis, since several excellent reviews have been recently published on this 
topic [36-38]. Instead, we will focus on the endocytic delivery of various regulatory proteins and their roles during abscission.

\section{Endosomes regulate the actin cytoskeleton within the ICB}

The actin cytoskeleton plays a key role during the initial formation and ingression of the cleavage furrow, as it acts with non-muscle myosin-II to form an actomyosin contractile ring [2]. Gradual constriction of this actomyosin ring leads to the formation of the ICB. Initially this ICB is stabilized by a cortical actin network, which is derived from the remnants of the actomyosin contractile ring. During late cytokinesis however, this cortical actin cytoskeleton must be disassembled to allow for abscission. One important actin cytoskeleton regulator is PtdIns $(4,5) \mathrm{P}_{2}$, which is produced de novo inside the ICB via the coordinated action of phosphatase (PTEN) and kinase (PI4K) (see Logan and Mandato [37], and Brill, JA. et al. [36] for further details). Interestingly, OCRL was recently identified as a Rab35-binding protein that is transported to the ICB by Rab35-endosomes [25]. OCRL is a $\operatorname{PtdIns}(4,5) \mathrm{P}_{2} 5$ phosphatase that was shown to induce actin cytoskeleton disassembly during interphase through the hydrolysis of PtdIns(4,5) $\mathrm{P}_{2}$ [39]. Consistent with the role of OCRL in abscission, depletion of either Rab35 or OCRL led to increased levels of PtdIns $(4,5) \mathrm{P}_{2}$ and F-actin within the ICB, as well as abscission defects [25].

Interestingly, Rab35 is not the only endocytic small GTPase that has been shown to regulate F-actin during cytokinesis, as both Rab11 and Arf6 regulate the actin cytoskeleton $[19,40,41]$. Work from Sullivan's laboratory has provided evidence that both Rab11 and its effector, Nuf (FIP3 orthologue in fly), are important in modulating actin polymerization in Drosophilia early embryos [19,40]. Mutations in either Rab11 or Nuf inhibit actomyosin contractile ring formation [19,40], suggesting a role for Rab11/Nuf in the initiation of Factin filament formation/assembly during cellularization in Drosophilia embryos. In contrast, in human cells, Rab11 or FIP3 depletion does not affect actomyosin ring formation or contraction [11,20,21]. Instead, Rab11 and FIP3 function at late cytokinesis, during the abscission of the ICB $[11,20,21,24]$. It was recently shown that FIP3-endosomes regulate the actin cytoskeleton during the division of mammalian cells by delivering Rho/Rac GTPase activating protein (GAP), p50RhoGAP [23]. When p50RhoGAP is knocked-down in HeLa cells, there is an increase in ICB cortical F-actin causing the delay in abscission [23]. During mammalian cell interphase, Rab11/FIP3 endosomes play a role in cell motility by regulating actin cytoskeleton dynamics within lamellipodia [42]. Furthermore, Arf6, a known FIP3binding protein, has been revealed to regulate the actin cytoskeleton, at least in part, by activating phosthatidlinositol-4-phosphate-5-kinase to produce $\operatorname{PtdIns}(4,5) \mathrm{P}_{2}$ [43]. Taken together, it is likely that Rab11, Rab35 and Arf6 cooperate to regulate the actin cytoskeleton inside the ICB during the final events leading to abscission.

\section{Endosomes and the ESCRT complexes during the abscission step of cytokinesis}

The contraction of the actomyosin ring leads to the formation of the ICB, a structure about $1-3 \mu \mathrm{m}$ in width that connects two daughter cells. The resolution of this ICB is the final abscission step of cytokinesis. Recent high-resolution tomography analyses have demonstrated that the abscission event is preceded by a narrowing of the ICB to $\sim 100 \mathrm{~nm}$, a step that is known as the secondary ingression and regulated by FIP3-endosomes 
$[11,23,30,44]$. Formation of this secondary ingression is required for abscission, and the mechanisms responsible for this localized ICB thinning are beginning to be revealed. Several years ago it was shown that the ESCRT protein complex is required for abscission, presumably by driving the final scission step in a manner similar to the scission of intraluminal vesicles during multi-vesicular body (MVB) formation, and the budding of enveloped viruses [45-47]. The sequential recruitment of specific ESCRT factors and their proposed function in cytokinesis have been covered in depth elsewhere [44,48]. Briefly, CEP55 is recruited to the midbody early during cytokinesis, shortly after the primary actomyosin-dependent ingression of the plasma membrane, followed by the arrival of Alix and Tsg101 (ESCRT-I) [44]. As the ICB matures, various ESCRT-III components, including CHMP4B, are also recruited to the midbody by binding to the CEP55/Alix/Tsg101 protein complex [44]. During the formation of MVB intraluminal vesicles, polymerization of CHMP4B monomers drives the scission event by constricting the bud neck and bringing its membranes into close proximity for fusion [30,44]. Recently, it was discovered that $17-$ $\mathrm{nm}$ filaments are present within the ICB, and that formation of these filaments is dependent upon CHMP2A (ESCRT-III) [30]. This observation led to the suggestion that the final constriction and abscission of the $1-3 \mu \mathrm{m}$ ICB is mediated by the formation of a continuous ESCRT-III oligomer from the midbody to the abscission site [30]. Recent studies using structured illumination microscopy also demonstrated the sequential association of various ESCRT proteins at the midbody, as well as movement of CHMP4B to the abscission site [44]. Interestingly, a continuous CHMP4B oligomer emanating from the midbody to the abscission site was not observed in these studies [44]. Instead, a disassociated pool of CHMP4B was shown to accumulate at the abscission site [44]. A question that arose from these studies is whether the ESCRT-III complex is actually involved in the formation of the secondary ingression. This question was recently addressed in HeLa cells, where the initiation and formation of the secondary ingression was shown to be dependent upon FIP3endosome fusion to narrow the ICB to $\sim 100 \mathrm{~nm}$, which is then subsequently stabilized by ESCRT-III to complete abscission (Figure 1). This model is further supported by the fact that the ESCRT-III complex preferentially associates with high curvature membranes $(>100$ $\mathrm{nm}$ ) in vitro [49], and is known to sever the narrow 20-50 nm necks of vesicles during MVB biogenesis and viral budding [50-53]. It has been shown that abscission often occurs only on one side of the ICB, thus resulting in one daughter cell inheriting the midbody [8,54]. Since recent studies have indicated that inheritance of the midbody can determine cell fate [54], it would be expected that the establishment of the abscission site is a highly regulated event during mitosis.

Recently it was observed that endosomes also play a role in determining the site of abscission through the initiation of the secondary ingression and possibly by recruiting ESCRT-III (Figure 1) [11,23]. Furthermore, FIP3-dependent delivery of SCAMP2/3 and p50RhoGAP, along with Rab35-dependent delivery of OCRL, are required for the localized disassembly of the actin cytoskeleton within the ICB [25]. It is likely that disassembly of the cortical actin network is required for the formation of the actin-free zone within the ICB, thus leading to increased ICB plasma membrane dynamics (Figure 1). Endosomes potentially can also deliver the components of the ESCRT-III complex to the ICB, as PI3Pcontaining endosomes transport FYVE-CENT to the ICB of dividing cells [55]. While the 
function of FYVE-CENT during cell division remains unclear, it was demonstrated that FYVE-CENT forms a complex with TTC19 and CHMP4B [55], thus potentially regulating ESCRT-III assembly at the abscission site (Figure 1). Lastly, recent work has shown that FIP3-endosomes deliver SCAMP3 to the ICB. Since by SCAMP3 interacts with TSG101 [56], FIP3-endosomes may also directly play a role in positioning ESCRT-III at the abscission site. In summary, the delivery and fusion of various endosomes with the ICB plasma membrane are directly involved in disassembly of the actin cytoskeleton to form the secondary ingression and create a region of high membrane curvature to recruit ESCRT-III oligomers for the final scission event (Figure 1).

\section{Conclusions}

Current work from many laboratories have demonstrated that abscission is a very complex cellular event that involves coordinated changes in the actin and microtubule cytoskeleton, endocytic membrane transport, ESCRT complex assembly and changes in membrane composition/dynamics. While the requirement for endocytic membranes in cytokinesis has been firmly established, the functions and regulation of these endosomes are only beginning to be uncovered. Thus, the identification and characterization of cargo proteins contained within Rab11/FIP3 and Rab35 endosomes will provide insights into their functional significance. Furthermore, improved high-resolution techniques, combined with time-lapse imaging and appropriate temporal markers will enable the questions presented in this review to be addressed in future studies.

\section{Acknowledgments}

We apologize to our colleagues for not being able to cite all work related to cytokinesis due to the focused nature of this review and its requirement for brevity. We are grateful to Carly Willenborg and Dr. Michael McMurray (University of Colorado Denver) for reviewing this manuscript. Research in Dr. Prekeris laboratory is supported by the National Institute of Health (DK064380), Susan G. Komen Breast Cancer Research Foundation and the Cancer League of Colorado Foundation.

\section{References}

1. Baluska F, Menzel D, Barlow PW. Cytokinesis in plant and animal cells: endosomes 'shut the door'. Dev Biol. 2006; 294:1-10. [PubMed: 16580662]

2. Barr FA, Gruneberg U. Cytokinesis: placing and making the final cut. Cell. 2007; 131:847-860. [PubMed: 18045532]

3. Eggert US, Mitchison TJ, Field CM. Animal cytokinesis: from parts list to mechanisms. Annu Rev Biochem. 2006; 75:543-566. [PubMed: 16756502]

4. Glotzer M. The molecular requirements for cytokinesis. Science. 2005; 307:1735-1739. [PubMed: 15774750]

5. Pollard TD. Mechanics of cytokinesis in eukaryotes. Curr Opin Cell Biol. 2009

6. Pollard TD, Wu JQ. Understanding cytokinesis: lessons from fission yeast. Nat Rev Mol Cell Biol. 2010; 11:149-155. [PubMed: 20094054]

7. Scholey JM, Brust-Mascher I, Mogilner A. Cell division. Nature. 2003; 422:746-752. [PubMed: 12700768]

*8. Gromley A, Yeaman C, Rosa J, Redick S, Chen CT, Mirabelle S, Guha M, Sillibourne J, Doxsey SJ. Centriolin anchoring of exocyst and SNARE complexes at the midbody is required for secretory-vesicle-mediated abscission. Cell. 2005; 123:75-87. Established a role for vesicle fusion during the final event of cytokinesis through centriolin interactions with the Exocyst 
complex and v-SNAREs. Also, demonstrates asymmetric delivery to the ICB during abscission. [PubMed: 16213214]

9. Kouranti I, Sachse M, Arouche N, Goud B, Echard A. Rab35 regulates an endocytic recycling pathway essential for the terminal steps of cytokinesis. Curr Biol. 2006; 16:1719-1725. [PubMed: 16950109]

10. Pohl C, Jentsch S. Final stages of cytokinesis and midbody ring formation are controlled by BRUCE. Cell. 2008; 132:832-845. [PubMed: 18329369]

**11. Schiel JA, Park K, Morphew MK, Reid E, Hoenger A, Prekeris R. Endocytic membrane fusion and buckling-induced microtubule severing mediate cell abscission. J Cell Sci. 2011; 124:14111424. Establishes that secondary ingression formation is required for and precedes the abscission event. Demonstrates that FIP3/Rab11 containing recycling endosomes contribute to the generation of the secondary ingression. One of three papers to recently investigate cytokinetic abscission using high-resolution imaging (also Ref30** and Ref44**). [PubMed: 21486954]

12. Donaldson JG, Jackson CL. Regulators and effectors of the ARF GTPases. Curr Opin Cell Biol. 2000; 12:475-482. [PubMed: 10873831]

13. Skop AR, Bergmann D, Mohler WA, White JG. Completion of cytokinesis in C. elegans requires a brefeldin A-sensitive membrane accumulation at the cleavage furrow apex. Curr Biol. 2001; 11:735-746. [PubMed: 11378383]

14. Farkas RM, Giansanti MG, Gatti M, Fuller MT. The Drosophila Cog5 homologue is required for cytokinesis, cell elongation, and assembly of specialized Golgi architecture during spermatogenesis. Mol Biol Cell. 2003; 14:190-200. [PubMed: 12529436]

15. Goss JW, Toomre DK. Both daughter cells traffic and exocytose membrane at the cleavage furrow during mammalian cytokinesis. J Cell Biol. 2008; 181:1047-1054. [PubMed: 18573914]

16. Shuster CB, Burgess DR. Targeted new membrane addition in the cleavage furrow is a late, separate event in cytokinesis. Proc Natl Acad Sci U S A. 2002; 99:3633-3638. [PubMed: 11891298]

17. Bukoreshtliev NV, Hodneland E, Eichler TW, Eifart P, Rustom A, Gerdes HH. Partitioning and Exocytosis of Secretory Granules during Division of PC12 Cells. Int J Cell Biol. 2012; 2012:805295. [PubMed: 22719766]

18. Pelissier A, Chauvin JP, Lecuit T. Trafficking through Rab11 endosomes is required for cellularization during Drosophila embryogenesis. Curr Biol. 2003; 13:1848-1857. [PubMed: 14588240]

19. Riggs B, Rothwell W, Mische S, Hickson GR, Matheson J, Hays TS, Gould GW, Sullivan W. Actin cytoskeleton remodeling during early Drosophila furrow formation requires recycling endosomal components Nuclear-fallout and Rab11. J Cell Biol. 2003; 163:143-154. [PubMed: 14530382]

20. Wilson GM, Fielding AB, Simon GC, Yu X, Andrews PD, Hames RS, Frey AM, Peden AA, Gould GW, Prekeris R. The FIP3-Rab11 protein complex regulates recycling endosome targeting to the cleavage furrow during late cytokinesis. Mol Biol Cell. 2005; 16:849-860. [PubMed: 15601896]

21. Fielding AB, Schonteich E, Matheson J, Wilson G, Yu X, Hickson GR, Srivastava S, Baldwin SA, Prekeris R, Gould GW. Rab11-FIP3 and FIP4 interact with Arf6 and the exocyst to control membrane traffic in cytokinesis. Embo J. 2005; 24:3389-3399. [PubMed: 16148947]

22. Hickson GR, Matheson J, Riggs B, Maier VH, Fielding AB, Prekeris R, Sullivan W, Barr FA, Gould GW. Arfophilins are dual Arf/Rab 11 binding proteins that regulate recycling endosome distribution and are related to Drosophila nuclear fallout. Mol Biol Cell. 2003; 14:2908-2920. [PubMed: 12857874]

**23. Schiel JA, Simon GC, Zaharris C, Weisz J, Castle D, Wu CC, Prekeris R. FIP3-endosomedependent formation of the secondary ingression mediates ESCRT-III recruitment during cytokinesis. Nat Cell Biol. 2012; 14:1068-1078. Showed that the FIP3-endosome delivery to the cleavage furrow mediates actin dissasembly, secondary ingression formation and ESCRT-III mediated abscission. [PubMed: 23000966] 
24. Simon GC, Schonteich E, Wu CC, Piekny A, Ekiert D, Yu X, Gould GW, Glotzer M, Prekeris R. Sequential Cyk-4 binding to ECT2 and FIP3 regulates cleavage furrow ingression and abscission during cytokinesis. Embo J. 2008; 27:1791-1803. [PubMed: 18511905]

*25. Dambournet D, Machicoane M, Chesneau L, Sachse M, Rocancourt M, El Marjou A, Formstecher E, Salomon R, Goud B, Echard A. Rab35 GTPase and OCRL phosphatase remodel lipids and F-actin for successful cytokinesis. Nat Cell Biol. 2011; 13:981-988. Demonstrated that the interaction between Rab35 and its effector OCRL, lead to phosphatidylinositol-4,5bisphosphate hydrolysis and F-actin depolymerization in the intracellular bridge. [PubMed: 21706022]

26. Dyer N, Rebollo E, Dominguez P, Elkhatib N, Chavrier P, Daviet L, Gonzalez C, Gonzalez-Gaitan M. Spermatocyte cytokinesis requires rapid membrane addition mediated by ARF6 on central spindle recycling endosomes. Development. 2007; 134:4437-4447. [PubMed: 18039970]

27. Kelly EE, Horgan CP, Adams C, Patzer TM, Ni Shuilleabhain DM, Norman JC, McCaffrey MW. Class I Rab11-family interacting proteins are binding targets for the Rab14 GTPase. Biol Cell. 2010; 102:51-62. [PubMed: 19702578]

28. Pellinen T, Tuomi S, Arjonen A, Wolf M, Edgren H, Meyer H, Grosse R, Kitzing T, Rantala JK, Kallioniemi O, et al. Integrin trafficking regulated by Rab21 is necessary for cytokinesis. Dev Cell. 2008; 15:371-385. [PubMed: 18804435]

29. Kaplan A, Reiner O. Linking cytoplasmic dynein and transport of Rab8 vesicles to the midbody during cytokinesis by the doublecortin domain-containing 5 protein. J Cell Sci. 2011; 124:39894000. [PubMed: 22159412]

**30. Guizetti J, Schermelleh L, Mantler J, Maar S, Poser I, Leonhardt H, Muller-Reichert T, Gerlich DW. Cortical constriction during abscission involves helices of ESCRT-III-dependent filaments. Science. 2011; 331:1616-1620. Demonstrates the formation of CHMP2-dependent 17nm-size filaments within the intracellular bridge during abscission. Suggests that these filaments are ESCRT-III oligomers. One of three papers to recently investigate cytokinetic abscission using high-resolution imaging (also Ref11** and Ref44**). [PubMed: 21310966]

31. Boucrot E, Kirchhausen T. Endosomal recycling controls plasma membrane area during mitosis. Proc Natl Acad Sci U S A. 2007; 104:7939-7944. [PubMed: 17483462]

32. Low SH, Li X, Miura M, Kudo N, Quinones B, Weimbs T. Syntaxin 2 and endobrevin are required for the terminal step of cytokinesis in mammalian cells. Dev Cell. 2003; 4:753-759. [PubMed: 12737809]

33. Lecuit T, Wieschaus E. Polarized insertion of new membrane from a cytoplasmic reservoir during cleavage of the Drosophila embryo. J Cell Biol. 2000; 150:849-860. [PubMed: 10953008]

34. Li WM, Webb SE, Chan CM, Miller AL. Multiple roles of the furrow deepening Ca2+ transient during cytokinesis in zebrafish embryos. Dev Biol. 2008; 316:228-248. [PubMed: 18313658]

35. Li WM, Webb SE, Lee KW, Miller AL. Recruitment and SNARE-mediated fusion of vesicles in furrow membrane remodeling during cytokinesis in zebrafish embryos. Exp Cell Res. 2006; 312:3260-3275. [PubMed: 16876784]

36. Brill JA, Wong R, Wilde A. Phosphoinositide function in cytokinesis. Curr Biol. 2011; 21:R930934. [PubMed: 22115464]

37. Logan MR, Mandato CA. Regulation of the actin cytoskeleton by PIP2 in cytokinesis. Biol Cell. 2006; 98:377-388. [PubMed: 16704377]

38. Nezis IP, Sagona AP, Schink KO, Stenmark H. Divide and ProsPer. the emerging role of PtdIns3P in cytokinesis. Trends Cell Biol. 2010; 20:642-649. [PubMed: 20880709]

39. Suchy SF, Nussbaum RL. The deficiency of PIP2 5-phosphatase in Lowe syndrome affects actin polymerization. Am J Hum Genet. 2002; 71:1420-1427. [PubMed: 12428211]

40. Cao J, Albertson R, Riggs B, Field CM, Sullivan W. Nuf, a Rab11 effector, maintains cytokinetic furrow integrity by promoting local actin polymerization. J Cell Biol. 2008; 182:301-313. [PubMed: 18644888]

41. Honda A, Nogami M, Yokozeki T, Yamazaki M, Nakamura H, Watanabe H, Kawamoto K, Nakayama K, Morris AJ, Frohman MA, et al. Phosphatidylinositol 4-phosphate 5-kinase alpha is a downstream effector of the small G protein ARF6 in membrane ruffle formation. Cell. 1999; 99:521-532. [PubMed: 10589680] 
42. Jing J, Tarbutton E, Wilson G, Prekeris R. Rab11-FIP3 is a Rab11-binding protein that regulates breast cancer cell motility by modulating the actin cytoskeleton. Eur J Cell Biol. 2009; 88:325341. [PubMed: 19327867]

43. Paleotti O, Macia E, Luton F, Klein S, Partisani M, Chardin P, Kirchhausen T, Franco M. The small G-protein Arf6GTP recruits the AP-2 adaptor complex to membranes. J Biol Chem. 2005; 280:21661-21666. [PubMed: 15802264]

**44. Elia N, Sougrat R, Spurlin TA, Hurley JH, Lippincott-Schwartz J. Dynamics of endosomal sorting complex required for transport (ESCRT) machinery during cytokinesis and its role in abscission. Proc Natl Acad Sci U S A. 2011; 108:4846-4851. Employed structured illumination and live cell microscopy to demonstrate the requirement of ESCRT-III complex formation at the midbody. Demonstrates that abscission is preceded by the recruitment of CHMP4B to the abscission site. One of three papers to recently investigate cytokinetic abscission using highresolution imaging (also Ref11** and Ref30**). [PubMed: 21383202]

45. Carlton JG, Agromayor M, Martin-Serrano J. Differential requirements for Alix and ESCRT-III in cytokinesis and HIV-1 release. Proc Natl Acad Sci U S A. 2008; 105:10541-10546. [PubMed: 18641129]

*46. Carlton JG, Martin-Serrano J. Parallels between cytokinesis and retroviral budding: a role for the ESCRT machinery. Science. 2007; 316:1908-1912. Showed for the first time the requirement for the ESCRT complexes for abscission. [PubMed: 17556548]

47. McCullough J, Fisher RD, Whitby FG, Sundquist WI, Hill CP. ALIX-CHMP4 interactions in the human ESCRT pathway. Proc Natl Acad Sci U S A. 2008; 105:7687-7691. [PubMed: 18511562]

48. Guizetti J, Gerlich DW. ESCRT-III polymers in membrane neck constriction. Trends Cell Biol. 2012; 22:133-140. [PubMed: 22240455]

49. Fyfe I, Schuh AL, Edwardson JM, Audhya A. Association of the Endosomal Sorting Complex ESCRT-II with the Vps20 Subunit of ESCRT-III Generates a Curvature-sensitive Complex Capable of Nucleating ESCRT-III Filaments. J Biol Chem. 2011; 286:34262-34270. [PubMed: 21835927]

50. Hanson PI, Roth R, Lin Y, Heuser JE. Plasma membrane deformation by circular arrays of ESCRT-III protein filaments. J Cell Biol. 2008; 180:389-402. [PubMed: 18209100]

51. Lata S, Schoehn G, Jain A, Pires R, Piehler J, Gottlinger HG, Weissenhorn W. Helical structures of ESCRT-III are disassembled by VPS4. Science. 2008; 321:1354-1357. [PubMed: 18687924]

52. von Schwedler UK, Stuchell M, Muller B, Ward DM, Chung HY, Morita E, Wang HE, Davis T, He GP, Cimbora DM, et al. The protein network of HIV budding. Cell. 2003; 114:701-713. [PubMed: 14505570]

53. Wemmer M, Azmi I, West M, Davies B, Katzmann D, Odorizzi G. Bro1 binding to Snf7 regulates ESCRT-III membrane scission activity in yeast. J Cell Biol. 2011; 192:295-306. [PubMed: 21263029]

*54. Kuo TC, Doxsey S. Fates and roles of post-mitotic midbodies beyond cytokinesis. Cell Cycle. $2012 ; 11: 7-8$. Discovered that midbody remnants are inherited asymmetrically to, and accumulate in, undifferentiated cell types such as stem cells and potentially cancer "stem cells.". [PubMed: 22157094]

*55. Sagona AP, Nezis IP, Pedersen NM, Liestol K, Poulton J, Rusten TE, Skotheim RI, Raiborg C, Stenmark H. PtdIns(3)P controls cytokinesis through KIF13A-mediated recruitment of FYVECENT to the midbody. Nat Cell Biol. 2010; 12:362-371. Identified a third class of endosomes that accumulates within the intracellular bridge during cytokinesis and contains phosphatidylinositol-3-phosphate enriched membranes. These endosomes also contain FYVECENT (a centrosomal protein) and TTC19, which interact with CHMP4B. Establishes a connection between endosomes and CHMP4B. [PubMed: 20208530]

56. Aoh QL, Castle AM, Hubbard CH, Katsumata O, Castle JD. SCAMP3 negatively regulates epidermal growth factor receptor degradation and promotes receptor recycling. Mol Biol Cell. 2009; 20:1816-1832. [PubMed: 19158374] 


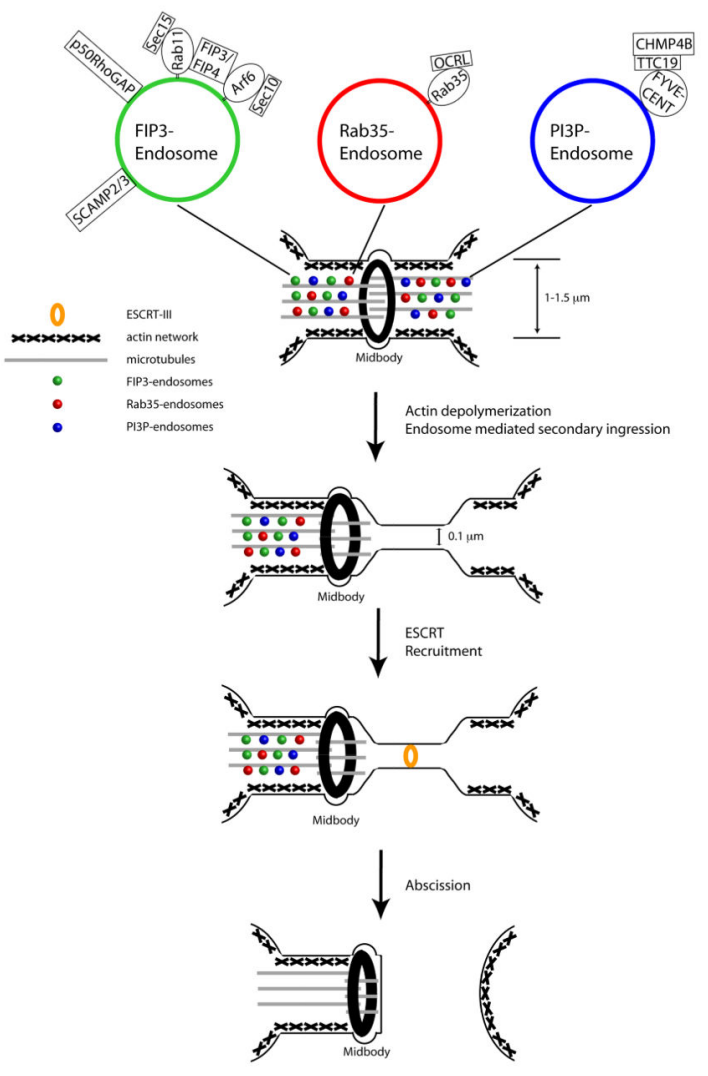

Figure 1. Endosome trafficking and ESCRT mediated scission of the ICB are required for the completion of cytokinesis

A model describing the two different mechanisms proposed to regulate the final abscission step in cytokinesis. In late cytokinesis, an actin stabilized intracellular bridge contains three types of endosomes that are known to localize to the intracellular bridge: FIP3-endosomes (Green), Rab35-endosomes (Red) or PI3P-enriched endosomes (Blue). Endosomes mediate ICB actin depolymerization and formation of the secondary ingression to narrow the bridge to $100 \mathrm{~nm}$. This thinner bridge now is recognized by CHMP4B which marks the abscission site and severs the plasma membrane to complete cytokinesis. The final membrane scission step by occurs through the action of CHMP4B (ESCRT-III). 
Table 1

Small GTPases and their effector proteins that localize to the intracellular bridge during cytokinesis

\begin{tabular}{l|l|l|c}
\hline Small GTPase & Organelle & Effector Protein (Cytokinesis) & REF \\
\hline Rab4 & Endosome & $?$ & 25 \\
Rab8 & Secretory/Endosome & $?$ & 10,28 \\
Rab11 & Recycling Endosome & FIP3 & $11,20-23$ \\
& & FIP4 & $20-22$ \\
& & Sec15 (Exocyst) & 8,21 \\
Rab21 & Recycling Endosome & $?$ & 27 \\
Rab35 & Endosome & OCRL & 9,24 \\
Arf6 & Endosome & JIP3 & 55 \\
& & JIP4 & 55 \\
& & FIP3 & $20-22$ \\
& & FIP4 & $20-22$ \\
& & Sec10 (Exocyst) & 8,21 \\
RalA/RalB & Endosome & Sec5/Exo84 (Exocyst) & 56,57 \\
\hline
\end{tabular}

